\title{
Saúde Pública ao Alcance da População: - Papel das Bibliotecas Virtuais nas Rádios Comunitárias
}

\author{
Public Health Reaching Undeserved People: the Role of \\ Virtual Libraries and Community Radios
}

\section{RESUMO}

Este trabalho tem o objetivo de apresentar a experiência de transferência da informação científica em saúde pública para comunidades carentes com o uso de bibliotecas virtuais para produção e veiculação de programas (spots) de rádio. A partir das teses e dissertações produzidas na Faculdade de Saúde Pública da Universidade de São Paulo (FSP-USP), os autores gravam programas de rádio, em linguagem jornalística, no seu Centro de Produção Digital. A Biblioteca: Centro de Informação e Referência (CIR) da FSP atua em duas frentes: indexação dos programas e disponibilização em bases de dados e bibliotecas virtuais a partir da tecnologia desenvolvida pela BIREME e qualificação dos comunicadores de rádios comunitárias para usarem as bibliotecas virtuais. Reforça-se a importância da integração de múltiplos profissionais, o papel do bibliotecário na organização da informação e qualificação dos comunicadores de rádio, do jornalista para a tradução do saber científico de forma correta e do comunicador de rádio na divulgação dos conteúdos.

Palavras-chave: Saúde pública. Bibliotecas virtuais. Rádios comunitárias.

\section{ABSTRACT}

This paper presents the experience in transferring scientific information in public heath for undeserved communities through the virtual libraries. This can be possible by production and transmission of spots in the radio schedule. The authors of the theses edited by the School of Public Health at University of São Paulo (FSP-USP) record spots with journalistic language in an Laboratory (Laudio) of the School. The Library: Reference and Information Centre operates in two fronts: the spots are indexed and available in databases and virtual library through the technology developed by BIREME. The Library promotes training to teach the broadcaster in how to access and how to use the virtual library. It emphasizes the importance of integration of multiple professional 
- librarian's role in information organization and teaching of broadcasters, the journalist for the translation of scientific knowledge accurately and the broadcaster to distribute this content to the undeserved community.

Keywords: Public health. Virtual libraries. Community radios.

\section{INTRODUÇÃO}

\section{O saber científico para beneficiar a sociedade deve ser compartilhado entre} os pesquisadores, comunicado em publicações e traduzido para a população. Na saúde pública, a transferência da informação para a população pode levar a benefícios imediatos, visto que sua produção não somente é baseada nas condições de vida da população como sua aplicação se reflete na saúde de grande número de pessoas.

Embora pareça óbvio, há grande dificuldade na disseminação das ações em saúde pública para comunidades pobres que devem ser o foco da atenção dos governos no que tange à saúde. A grande questão é: como a gestão descentralizada do conhecimento científico pode alcançar comunidades carentes e estas se beneficiarem das informações e descobertas em saúde pública? Algumas iniciativas em âmbito local [1] e internacional [2] vêm sendo implementadas por setores sociais e governamentais, e inseridas nas políticas públicas visando ampliar as ações voltadas à integração da população no universo da cidadania [6] .

Como as rádios comunitárias são veículos potenciais de penetração nas comunidades carentes e seus comunicadores contam com grande credibilidade da população local, a iniciativa de veiculação via rádio de conhecimentos de saúde pública produzidos no âmbito universitário foi oficialmente referendada na Reunião da Cúpula Mundial sobre a Sociedade da Informação, em Genebra, 2003. Nessa reunião, foram firmados compromissos de empenho dos países para a redução do hiato de conhecimento entre as classes sociais e para a minimização dos problemas da exclusão digital, do acesso à internet e do direito à comunicação [8].

No entanto, no caso brasileiro, foi constatado que, por vezes, as informações em saúde veiculadas em programações de rádios comunitárias mostravam-se confusas ou distorcidas dos conteúdos originais [6]. Disto surgiu a ideia de qualificar os comunicadores de rádios em noções de saúde pública e, também, no uso de bibliotecas virtuais para acesso à informação.

Este trabalho tem o objetivo de apresentar o relato da experiência de disseminação da informação científica em saúde pública a comunidades carentes com o uso de bibliotecas virtuais para a produção e veiculação de programas de rádio em acesso aberto na internet.

\section{A IDEIA}

Após alguns cursos de noções de saúde pública para comunicadores de rádios comunitárias de bairros pobres da Grande São Paulo, que incluíam o aprendizado do acesso e uso de bibliotecas virtuais, surgiu a ideia de se criar uma fonte de informação, neste caso, uma série de programas de rádio que ficariam disponíveis numa biblioteca virtual. 
Biblioteca virtual é a expressão usada quando se extrapola o conceito de biblioteca física, implicando um espaço virtual na internet onde a informação científica e técnica, validada por especialistas, é registrada, organizada e armazenada em formato eletrônico e acessível de forma universal. Esse conceito permeia a ideia de acesso imediato e gratuito aos conteúdos [7], ou seja, os programas de rádio seriam organizados de forma que, qualquer pessoa, radialista, agente comunitário, entre outros interessados, pudessem ter acesso em qualquer hora e de qualquer computador ligado à internet.

Para serem inseridas em bibliotecas virtuais, as informações científicas seriam traduzidas para uma linguagem jornalística e tornar-se-iam presentes na grade de programação das rádios comunitárias em formato adequado à característica de cada comunidade. Os agentes comunicadores, geralmente líderes comunitários, seriam qualificados para o acesso e uso das bibliotecas virtuais e, desta forma, eles próprios poderiam selecionar a informação e reeditá-la de acordo com a necessidade e demanda sentidas em suas comunidades.

Para tornar essa ideia uma realidade, vários saberes técnicos e instituições teriam que ser mobilizados.

\section{O ESTABELECIMENTO DAS PARCERIAS}

A Faculdade de Saúde Pública da Universidade de São Paulo (FSP-USP), uma das maiores produtoras brasileiras de pesquisa em saúde pública, visando potencializar a divulgação de informações em saúde para a população, realizou vários cursos de extensão universitária para comunicadores de rádio, em parceria com o Ministério da Saúde e a Associação Brasileira de Rádios Comunitárias [6]. Esses cursos tiveram por objetivo qualificar os líderes comunitários em noções de saúde pública, na realização de diagnóstico comunitário de saúde e na produção de programas de rádio. Os participantes eram líderes comunitários (de várias regiões que compõem a chamada periferia da Grande São Paulo) e, em outra vertente, docentes (potenciais multiplicadores desse saber nas demais universidades brasileiras).

A Biblioteca: Centro de Informação e Referência (CIR) da FSP-USP, além de manter um acervo impresso de mais de 350 mil volumes, um dos maiores da América Latina em saúde pública e nutrição, é dotada de infraestrutura tecnológica e de equipes bibliotecárias especializadas na construção de bibliotecas virtuais [3] e na capacitação de usuários [4]. Por esse motivo, participa dos cursos para comunicadores de rádios comunitárias desenvolvendo módulos práticos de acesso e uso de bibliotecas virtuais para que possam contar com uma ferramenta de busca de informação com validade científica. A Biblioteca/CIR, além de conduzir sua versão de website e serviços on-line, também é parceira da Biblioteca Virtual em Saúde Pública, desenvolvida pela BIREME [7], portanto, com competência na metodologia e manutenção de bibliotecas virtuais.

A BIREME lidera a tecnologia para a disseminação da informação em saúde, cujas iniciativas exitosas - desde a década de 1980, como base de dados LILACS, a SciELO nos anos de 1990 e a tecnologia da Biblioteca Virtual em Saúde (BVS) nos anos de 2000 - vem contribuindo com a visibilidade da produção científica e a promoção da saúde no Brasil 
e países da América Latina. Essas bibliotecas virtuais operam integrando diversas fontes de informação, tais como: literatura técnica e científica produzida nos países; base de sites especializados; diretório de eventos; lista de especialistas na área; indicadores de saúde; textos completos de artigos científicos, de teses e monografias e de editoras eletrônicas.

A união das expertises dessas instituições culminou na construção de uma fonte de informação, neste caso, em um portal de áudio. Os pesquisadores gravam depoimentos e entrevistas sobre suas teses no Centro de Produção Digital da FSP-USP, que os transforma em programas (spots) de rádio. Estes são armazenados em servidores (repositório institucional da Biblioteca), organizados e descritos em seus metadados, em planilhas desenvolvidas na metodologia BIREME. Os arquivos são disponibilizados na base de dados LILACS, nos catálogos da Biblioteca (como Audioteca) e na Biblioteca Virtual em Saúde Pública (BVSP) via Portal de Teses e Dissertações.

* No site da Biblioteca da FSPUSP (www.biblioteca.fsp.usp. br), em Bibliotecas Virtuais, selecionar Audioteca e digitar na caixa de busca o assunto desejado (meio ambiente, por exemplo). Clicando no ícone de áudio você poderá ouvir o spot de rádio. Caso queira ver a lista de todos os spots basta digitar \$ (cifrão) na caixa de busca e <enter>.

** No site da Biblioteca Virtual em Saúde/BIREME (www. bvs.bireme.br) selecionar

Literatura Científica e digitar na caixa de busca o assunto desejado (meio ambiente, por exemplo) e clicar Pesquisa. Na coluna à direita em Filtrar, selecionar no final da tela, em Tipo de Documento o item Áudio. Aparecerá a lista dos registros referentes às teses defendidas na FSP-USP com programas de áudio. São dois links: um com o spot

sobre a tese e outro com uma entrevista com o autor.

\section{O RESULTADO}

Para ampliar as possibilidades de acesso ao conhecimento, foram inseridas novas mídias como fontes de informação no campo da saúde pública em uma interface em áudio organizada em uma Audioteca. Seu acervo é composto por registros de depoimentos de pesquisadores a respeito de suas dissertações [5]. Participam dessa iniciativa pesquisadores, docentes, bibliotecários, jornalistas e pessoal de informática. Essa nova fonte de informação resulta da parceria de várias instituições que disponibilizam seus arquivos em áudio nas suas bibliotecas virtuais e, assim, se estabelece uma rede social.

Nessa experiência, foram produzidos, até 2013, cerca de 200 spots ou programas de rádio, com acesso universal e gratuito na internet via catálogos da Biblioteca da FSP-USP* e via base de dados LILACS**.

$\mathrm{Na}$ Figura 1 é possível visualizar um registro de tese nas bases da Biblioteca e na Figura 2, o registro na base de dados LILACS. Ambas com arquivos em áudio associados e respectivos ícones para reconhecimento do usuário. Cada ícone representa um arquivo extensão .mp3 com áudio de cerca de 6 minutos.

As mídias em áudio podem atender às demandas de informação de várias categorias de usuários: o pesquisador pode utilizá-las como objeto de investigação; um professor do Ensino Médio ou Fundamental para fundamentar suas aulas; serviços de saúde para auxiliar na prática de seus profissionais; os radiocomunicadores para contribuir para a promoção da saúde nas regiões em que estão inseridos.

Em breve, o número de acessos deverá ser aumentado quando a BIREME colocar para acesso público o Portal de Áudio, como uma das mais novas fontes de informação da Biblioteca Virtual. Outras instituições parceiras como a Fiocruz, por meio da Escola Nacional de Saúde Pública e o Ministério da Saúde, também deverão colocar seus acervos em áudio nesse Portal.

Achado o caminho, houve necessidade de desenvolver planilhas, ou seja, customizar o software, para a descrição de áudio e vídeo de outros tipos de documentos como livros, artigos, congressos, entre outros que disseminam a informação científica em saúde pública. 


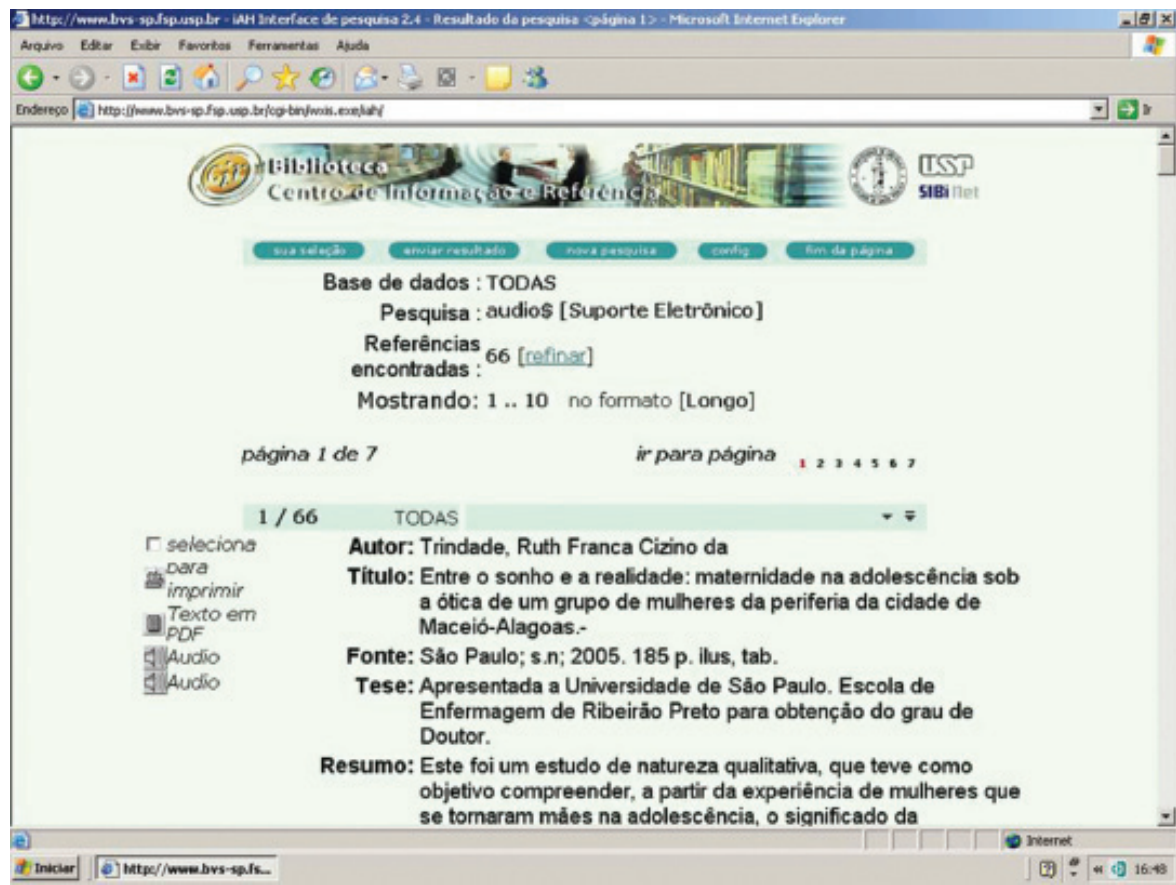

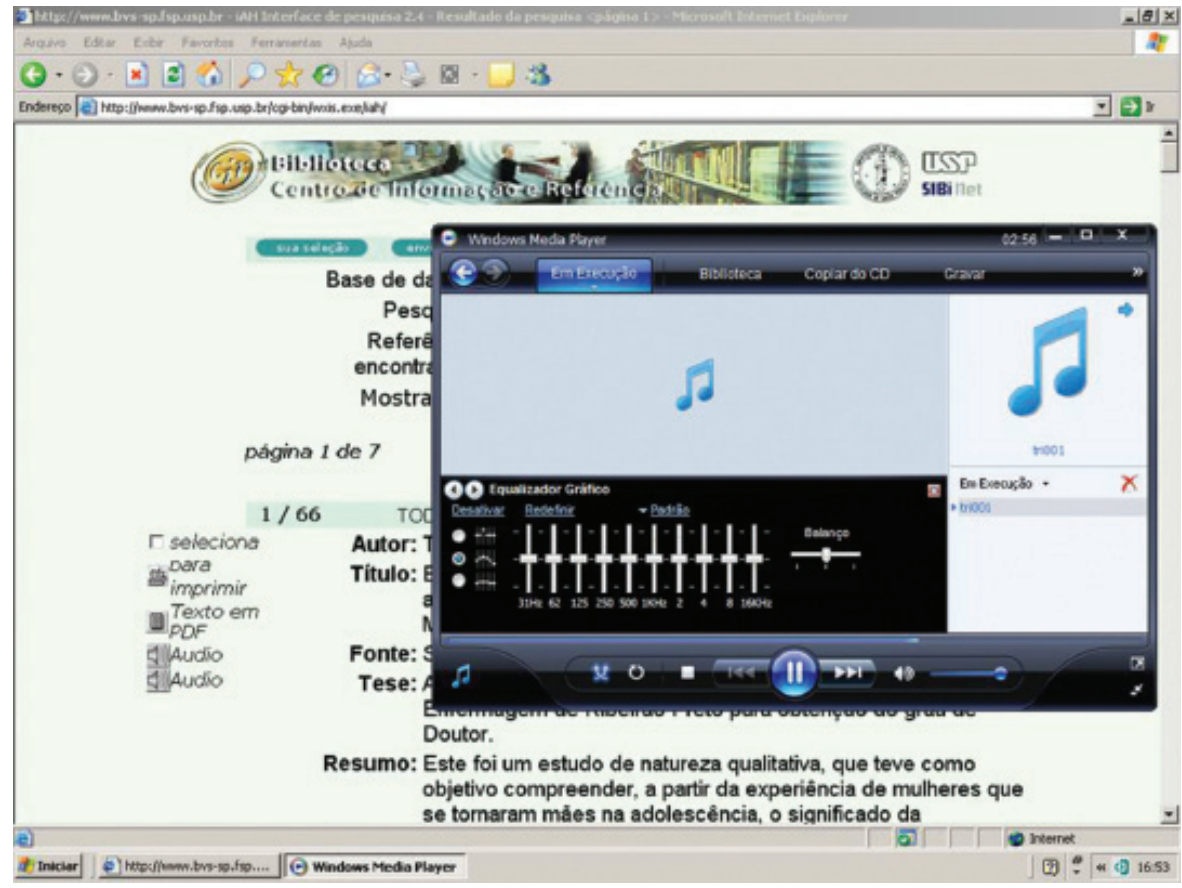

Figura 1 - Registro da tese na base de dados da Biblioteca com a indicação de áudio.
Figura 2 - Registro da tese na base de dados da Biblioteca durante a execução do áudio 
Para avaliação do impacto dos programas de rádios nas comunidades está sendo desenvolvido um estudo com as rádios comunitárias participantes do projeto sobre alterações sentidas nas ações de saúde, a percepção dos profissionais de saúde e gestores dessas regiões sobre o conhecimento da população dos direitos à saúde e, com os comunicadores de rádio, sobre as mudanças ocorridas antes e depois da inserção das noções de saúde nos cursos de difusão universitária e na programação das rádios.

\section{LIÇÕES APRENDIDAS}

A integração multidisciplinar de profissionais envolvidos, a intersetorialidade da produção do saber científico e a possibilidade de sua gestão de forma descentralizada permitem perceber a importância de um trabalho voltado à equidade das ações em saúde no âmbito das comunidades mais pobres.

O desenvolvimento dessa nova fonte de informação reuniu cientistas, jornalistas, produtores e comunicadores de áudio, bibliotecários, profissionais ligados à tecnologia da informação, webdesigners, analistas de sistemas, entre outros, convergindo para um mesmo fim, a transferência do conhecimento científico para a população, constituindo um aprendizado inigualável.

A importância do bibliotecário, principalmente das bibliotecas acadêmicas, mostra-se em sua atualização para acompanhar a necessidade do novo usuário, passando a ser o educador (trainer), qualificando o usuário a se tornar permanentemente autônomo para fazer a busca da informação, de forma eficiente e, sobretudo, eficaz. Desta forma, estabelece-se o aprendizado ao longo da vida, característica da competência informacional tão discutida nos dias atuais.

A participação de alunos de jornalismo nos grupos de pesquisa e de desenvolvimento de TI tem sido fundamental para que a linguagem científica torne-se acessível às populações carentes, algo imprescindível na comunicação via rádios comunitárias, criando-se condições para que a universidade experimente novas formas de extensão.

Essa experiência propicia forte incremento no processo de educação e promoção de saúde em âmbito comunitário, por meio da inclusão digital. Reforça-se, assim, a responsabilidade da transferência da informação científica, de forma correta, com responsabilidade social, para que esta tenha seu uso adequado, ou seja, transmitir uma informação com validade científica traduzida sem vieses, sem outras conotações que não sejam as de promover a saúde e reduzir a desigualdade social junto à população carente.

\section{REFERÊNCIAS}

[1] BIREME. $8^{\circ}$ CONGRESSO REGIONAL DE INFORMAÇÃO EM CIÊNCIAS DA SAÚDE (CRICS). Rio de Janeiro, 16-19 set. 2008. Declaração do Rio. Disponível em: <http://bvs5.crics8.org/php/index.php?lang=pt>. Acesso em: 2 ago. 2013.

[2] CORDEIRO, C. et al. Funding agencies in low-and middle-income countries: support for knowledge translation. Bull. WHO, Manila [Filipinas], v. 86, n. 7, p. 524-534, 2008. 
[3] CUENCA, A. M. B. et al. Biblioteca virtual e o acesso às informações científicas e acadêmicas. Revista USP, São Paulo, v. 80, p. 72-83, 2009.

[4] CUENCA, A. M. B. et al. Capacitação no uso das bases Medline e Lilacs: avaliação de conteúdo, estrutura e metodologia. Ciência da Informação, Brasília , v. 28, n. 3, dez. 1999. Disponível em: <http://www.scielo.br/scielo.php?script=sci arttext\&pid=So100-19651999000300012\&lng=pt\&nrm=iso $>$. Acesso em: 01 nov. 2013. http://dx.doi.org/10.1590/So100-19651999000300012.

[5] Delbucio, H. C. R. F.; Noronha, G. P.; Santos, C. A. M. dos. Inserção de mídia em áudio no site da Biblioteca/CIR da Faculdade de Saúde Pública da USP. In: $4^{\circ}$ SEMINÁRIO INTERNACIONAL DE BIBLIOTECAS DIGITAIS BRASIL (SIBDB), São Paulo: UNICAMP; 2007. Disponível em: <http://libdigi. unicamp.br/document/?view=23461>. Acesso em: 2 ago. 2013.

[6] GALLO, P. R. Radiofusão comunitária: um recurso a ser valorizado no âmbito da educação em saúde. Saúde em Debate, Rio de Janeiro, v. 25, p. 59, p. 59-66, 2001.

[7] PACKER, A. L. A construção coletiva da Biblioteca Virtual em Saúde. Interface - Comunicação, Saúde, Educação, Botucatu, v. 9, n.17, p. 249-72, 2005. Disponível em: < http://dx.doi.org/10.1590/S1414-32832005000200004>

[8] UNITED NATIONS. World Summit on the Information Society. WSIS Plan of action. Geneva, 2003. Disponível em: <http://www.itu.int/wsis/docs/geneva/ official/poa.htm> . Acesso em: 2 out. 2013

\section{AGRADECIMENTOS}

Agradecimentos à equipe multidisciplinar que vem atuando na fonte de informação $\mathrm{Au}-$ dioteca: as bibliotecárias Claudia Guzzo, Hálida C. R. Fernades, Eidi R. F. Abdalla, Maria Imaculada da Conceição; Maria do Carmo Avamilano Alvarez; as jornalistas Claudia Malinverni, Heloisa Ferraz; as especialistas em saúde pública Sophia K Motta-Gallo e Alina Zoqui Cayres; o técnico de áudio Amadeu dos Santos; na informática, Daniel Marucci e equipe, e a todos os pesquisadores que se propuseram a gravar os programas.

ANGela mARIA Belloni CUENCA docente do Departamento de Saúde Materno-Infantil da Faculdade de Saúde Pública da Universidade de São Paulo (FSP-USP) e coordenadora dos projetos da Biblioteca Virtual em Saúde Pública na Biblioteca/CIR da FSP-USP - e-mail: abcuenca@usp.br

PAUlO ROGÉRIO GALLO docente do Departamento de Saúde Materno-Infantil da Faculdade de Saúde Pública da Universidade de São Paulo (FSP-USP) e coordenador do Laboratório de Áudio da FSP-USP - e-mail: prgallo@usp.br 